\title{
SPILL STRUCTURE IN INTENSE BEAMS *
}

\author{
M Blaskiewicz, K Brown, JW Glenn, E Raka, J Ryan, \\ BNL, New York 11973, USA
}

\section{ABSTRACT}

Fixed target studies of small branching ratio decay processes require intense beams and smooth spills. Longitudinal structure arises through collective effects, well below the coasting beam stability threshold. These structures have been observed at the Brookhaven AGS and dependence on intensity and momentum spread measured. Measurements and amelioration techniques have been developed and will be described.

\section{INTRODUCTION}

The delivery of constant beam current to counter experiments at high intensities has been plagued by excessive noise like structure. Two unexpected phenomena have been noted: small clusters of protons remain as stable 'hot spots' for extended times; and the Keil-Shnell formula predicts an impedance 50 times larger than expected.

The AGS extraction relies on the chromaticity of the accelerator and an induced momentum spread in the beam This spread is $\sim 1 / 2 \%$ delP/P and the field is lowered by this amount over the two seconds of flattop to spiral the debunched beam into a third integer resonance. Spill ripple initially was dominated by correction supplies that modulated the betatron tune. This was nearly eliminated with the installation of low ripple power supplies

With intensity increases in the AGS spill ripple again increased. This is due to collective effects in the spiraling beam awaiting extraction. Measurement of the AGS is required to understand how these bunches behave or even exist. Also this affects the limit to coasting beam intensity, which will be pushed for proton operation of the AGS.

\section{SPILL MEASUREMENTS}

The external beam current at the AGS is measured by a secondary emission chamber with $3 \mathrm{kHz}$ electronics. The first two figures show how the structure increases disproportional with intensity. The first is at $2 \times 10^{13} \quad(20 \mathrm{TP})$ protons extracted from the AGS. The ripple in the current is steady and due to power supply ripple. The second shows large bursts of 'noise' in the spill. The spill bursts are fast as see in Fig 3, some only a few mSec. The average momentum swing over this period is less than the $5-7 \times 10^{-4}$ tune width of the extraction resonance times the chromiticity

*Work performed under Contract Number DE-AC0298CH10886 with the US Department of Energy
The density in momentum of these 'hot spots' is of order 5 times the average density of the coasting beam.

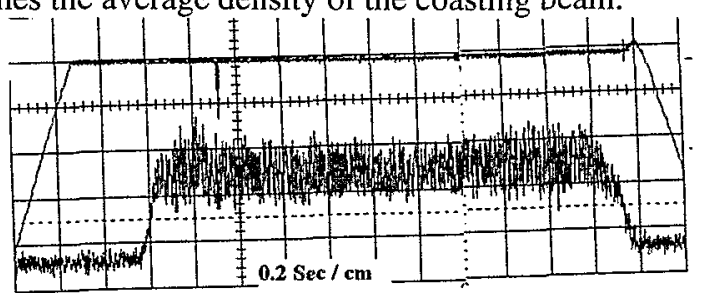

Fig 1. SEB Beam Current @ 20 TP in AGS

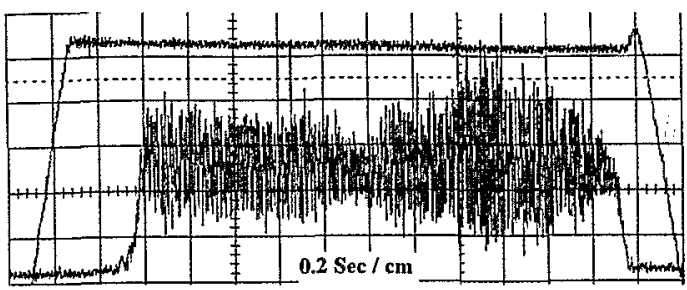

Fig 2. SEB Beam Current @ 50 TP in AGS
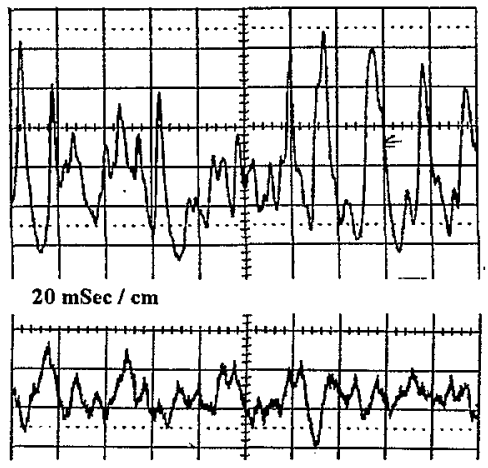

Fig 3 Fast Sweep of Spill Structure 50 \& 20 TP

That these bursts are not periodic is seen in the spectra of the current, at high intensity it is mostly a noise continuum not the series of harmonics as seen at lower intensity.

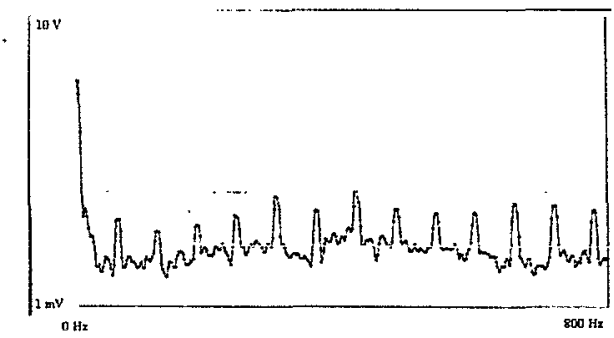

Fig 4 Spectrum of Spill Current - $20 \mathrm{TP} / 2 \mathrm{Sec}$ 


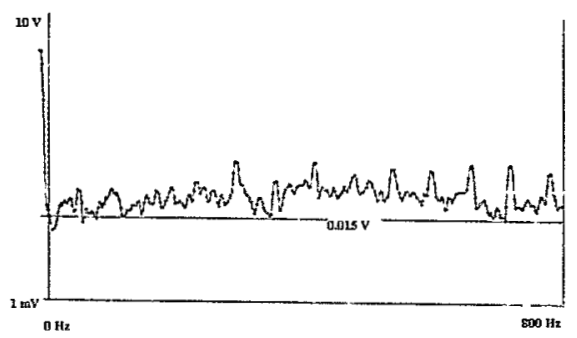

Fig 5 Spectrum of Spill Current - $50 \mathrm{TP} / 2 \mathrm{Sec}$

\section{COASTING STABLE BUNCHES}

These hotspots are also visible longitudinally on our fast ( $100 \mathrm{MHz})$ beam, or wall, current monitor. This display plots 100 sequential traces from the scope each 5 $\mathrm{mSec}$ later and plots them in front of the last as a contour map, 'mountain range', plot. Each trace is about one and a half AGS revolutions so the whole turn is shown. The beam is debunched on flat top by jumping the RF phase to the unstable fixed point and defocusing for a quarter synchrotron oscilation, then shutting down the RF. The plotting starts within a few $\mathrm{mSec}$ after this and continues into spill time. There are clusters of protons that remain stable for hundreds of $\mathrm{mSec}$. The way they interact is reminiscent of the 'holes' simulated and measured in the CERN PS Booster [1]. The theory of solitons in coasting beams in this paper predicts that hot spots should be associated with a focussing impedance. However AGS extraction is well above transition and previous measurements [2] suggest an inductive broad band $|\mathrm{Z} / \mathrm{n}| \approx$ $10 \Omega$. This leads to a defocusing impedance. The actual $\mathrm{Z} / \mathrm{n}$ is a function of frequency and our data suggests that the details of the frequency dependence are important.

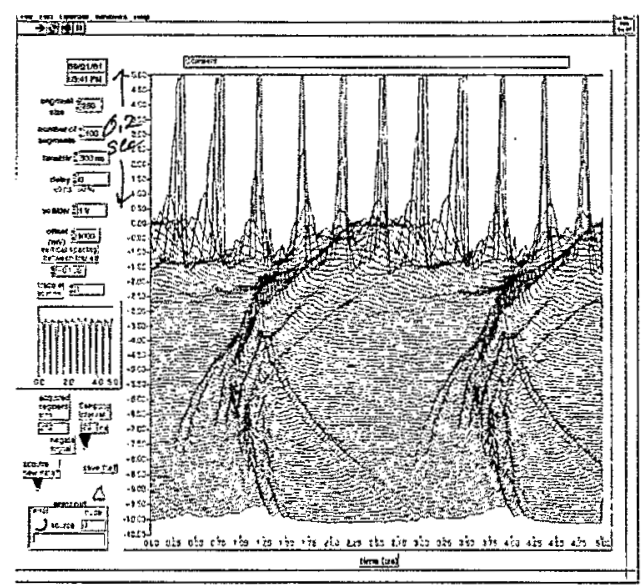

Fig 6 Lasting Bunches on Mountain Range

We studied how these bunches were effected by intensity in the AGS and the energy spread induced during RF turnoff. For a series of RF voltages at turn off, with and without the phase jump, we watched these bunches decay or not for different intensities. The current of the bunches was recorded.

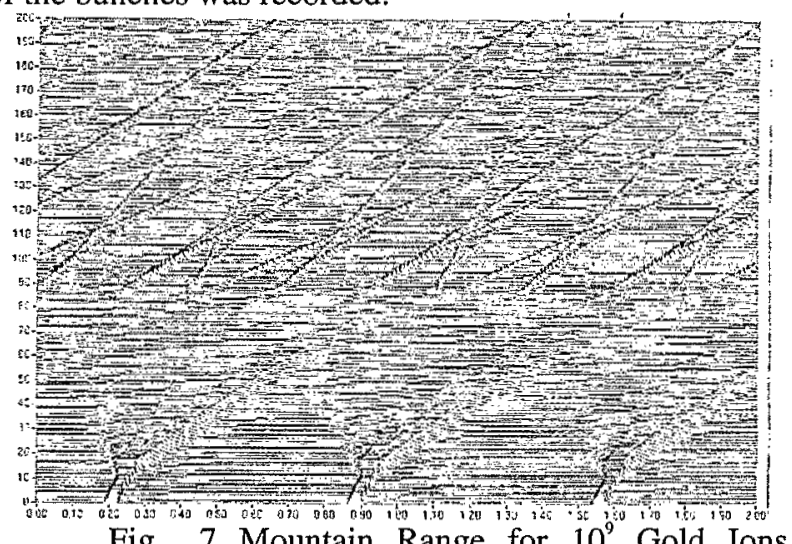

Fig 7 Mountain Range for $10^{9}$ Gold Ions. showing a picture of solitons of gold show and that high intensity is not essential for stable isolated clusters.

Also we measured the resultant momentum spread for various RF voltages at turnoff. Two methods were used as shown in Fig 7. First the difference in rotation frequency within an accelerated beam bunch was measured by noting how much the bunch widened over $50 \mathrm{mSec}$. Note the asymmetry of debuncing is similar to that modeled and seen in the CERN SPS [3]. The other method was to measure the time for the beam to be extracted with a constant rate of fall of the main magnet field. This time is determined by the tune, thus momentum, width of the beam

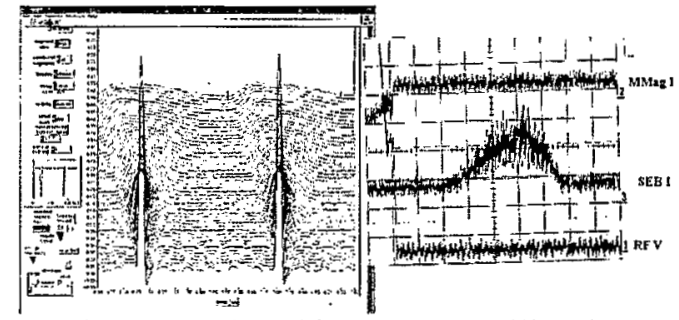

Fig 7 Debunching and Spill pictures for measurement.

Table I, Measured Momentum Spread

\begin{tabular}{ccc} 
RF Volt & \multicolumn{1}{c}{ DelP/P - \% } \\
kV & Debunch & Spill \\
& & \\
0 & 0.337 & 0.26 \\
8 & 0.422 & 0.35 \\
15 & 0.432 & 0.35 \\
60 & 0.48 & 0.41
\end{tabular}




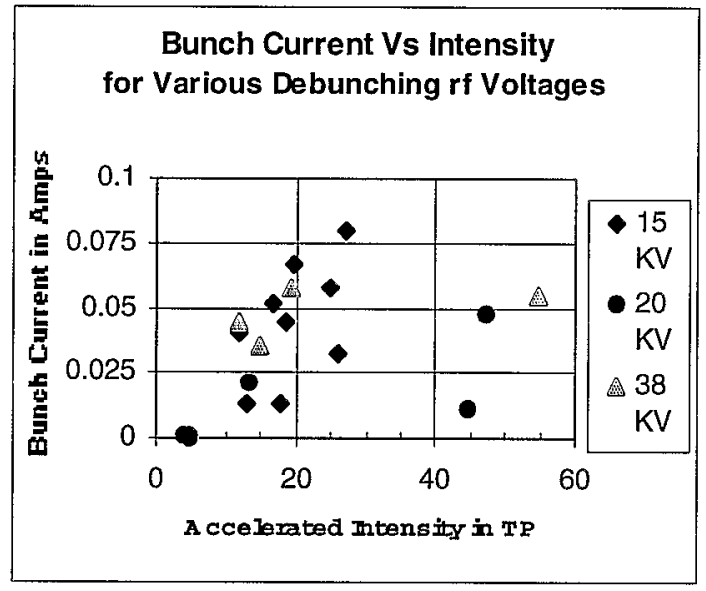

Fig 8 Beam Current of 'Hot Spots'

The results of this 'mapping' show that the primarily affector of hot spot size is the beam intensity during acceleration and the RF voltage at debunching has little effect,

$$
\left(\frac{\Delta P}{P}\right)^{2}=\frac{-4}{\pi} * \frac{(Z / n) \cdot \Delta I}{\eta *\left(E_{T} / q\right)}
$$

This is an extension Koscielniak's work [1] which gives a criteria for stable solitons For stable solitons to exist in the AGS above transition, with its $\eta=0.0116$ and an $\mathrm{E}_{\mathrm{T}} / \mathrm{q}=20 \mathrm{GeV}$ and using a broad band $\mathrm{Z} / \mathrm{n}=10 \Omega$, for $0.1 \mathrm{~A}$ of protons the delP/P would be $7.4 \times 10^{-5}$; and for the $2 \times 10^{9}$ Gold ions with a full delP/P of $\sim 2.5 \times 10^{4}$ the delP/P of a soliton of $10 \%$ ot the beam would have a delP/P of $10^{-5}$. We currently do not have the capacity to measure this but it may be possible using the SEB. This calculation assumes a negative inductance contrary to the above mentioned measurement.

\section{AGS IMPEDANCE}

While mapping the effects on the stable bunches during the spill, we also raised intensity with the RF voltage held constant and noted beam losses. The intensity and momentum spread that the beam goes unstable and is lost is a function of the impedance.

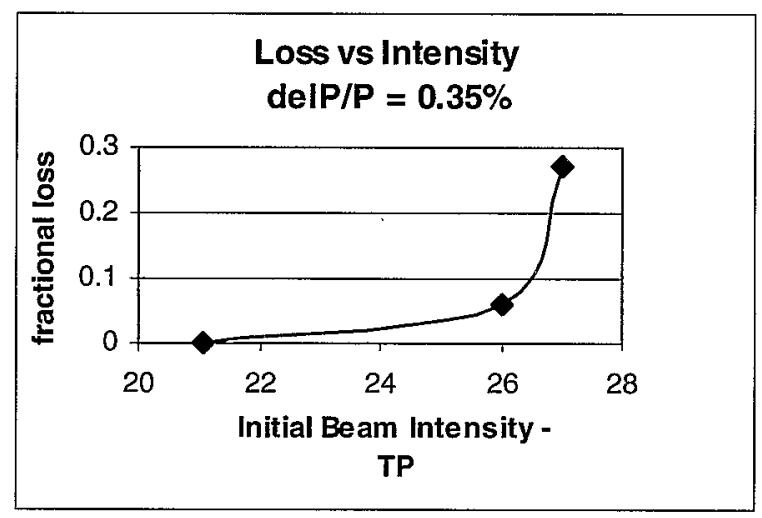

Fig 9 Beam Loss vs. Intensity, constant delP/P

Assuming that this behavior is the result of a coasting beam longitudinal instability and applying the
Keil-Schnell criteria [4], implies $[\mid \mathrm{Z} / \mathrm{n}\} \approx 550 \Omega$. Using this impedance, the expected limit of beam intensity vs. momentum spread and results from this test are plotted.

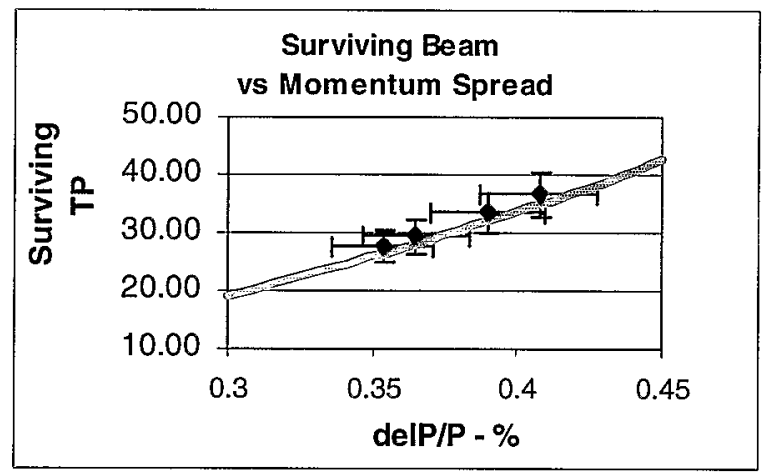

Fig 10 Surviving Beam Intensity vs. delP/P

This value is more than 50 times larger than previous estimates. We conclude that if the data in Fig 9 \& 10 are due to longitudinal effects, which is reasonable considering the quantity of 'seed' solitons, these effects cannot be understood within the context of linear stability theory. To achieve $100 \mathrm{TP}\left(10^{14}\right)$, a $0.7 \%$ delP/P would be necessary to stabilize the beam.

\section{AMELIORATION}

The VHF cavity continues to be our main stay to prevent bunches affecting the spill [5]. Whether it will continue to be effective at higher intensities is a concern.

\section{CONCLUSIONS}

The high impedance measured must be better understood. This is presumably why bunches are stable above transition, while it is expected with normal inductive impedance they would be unstable and disperse. Understanding and correcting or compensating for this impedance would have a great benefit on machine performance.

\section{REFERENCES}

[1] S Koscielniak, et.al, 'Longitudional Holes ..', Phys Rev Special topics - Accels \& Beams, Vol 4, 044201 (2001)

[2] F Pedersen, E Raka 'A Measurment of Longitudinal ..' PAC79, IEEE NS-26.\#3, June '79

[3] T Bohl, et.al ' Study of a Single Bunch Instability ..' EPAC 2000, CERN-SL-2000-019 HRF

[4] pp211, D Edwards \& $M$ Syphers 'An Introduction to the Physecs ...', J Wiley for discussion and reference.

[5] K Brown et.al, 'Status of Slow Extraction ...', TPPE003 this conference 